Article

\title{
A Study on the Abrasive Gels and the Application of Abrasive Flow Machining in Complex-Hole Polishing
}

\author{
A-Cheng Wang ${ }^{1, *}$, Ken-Chuan Cheng ${ }^{1}$, Kuan-Yu Chen ${ }^{2}$ and Yan-Cherng Lin ${ }^{3}$ \\ 1 Department of Mechanical Engineering, ChienHsin University of Science and Technology, Taoyuan 32097; \\ acwang@uch.edu.tw; kccheng@uch.edu.tw \\ 2 Department of Mechanical Engineering, Chung Yuan Christian University, Taoyuan 32023; \\ gychen@cycu.edu.tw; ccchien@cycu.edu.tw \\ 3 Department of Mechanical Engineering, Nankai University of Technology, Nantou 54243; \\ * Correspondence: acwang@uch.edu.tw; Tel.: +886-3-4581196 ext.5500
}

\begin{abstract}
Due to the fact that abrasive media can deform flexibly in abrasive flow machining (AFM) making this method easily to polish the complex holes and the curved surface of the hard machining shapes. Although abrasive media dominate the polishing behavior in AFM process, the mechanism of the abrasive media are not easy to understand because of the high viscous gels, therefore, many finishing works need lots of time to design AFM process. Power laws of the abrasive gels is studied here to evaluate the non-Newtonian flow of abrasive gels in complex holes polishing firstly, at the mean time different abrasive gels are utilized to finish the complex holes to follow the results by non-Newtonian flow calculation. Moreover, traditional AFM has difficulty to achieve a uniform roughness of radial distribution in the complex holes polishing because of the non-uniform abrasive forces. So a helical passageway is proposed to create a multiple motion of abrasive medium and to obtain the even surface of the complex holes in AFM process.
\end{abstract}

Keywords: abrasive flow machining; a helical passageway; surface roughness, mold core

\section{Introduction}

The benefits of AFM method consist of convenient operation, lower manufacturing cost, complex shape machining, and higher polishing efficiency. It is widely used to deburr, radius, polish, and remove recast layer of the complex geometries and hard materials. However, such as other non-conventional polishing processes, a smoother surface of workpiece is not easily obtained after AFM polishing, since this process had the constraint in the lower rates of material removal. To overcome different limitations of the traditional AFM process, there are many kinds of different skills and methods to be developed. By using AFM as a base process, a lot of hybrid polishing process like magnetic abrasive finishing, magneto rheological finishing, magnetic float polishing, elastic emission machining, etc. have been developed having component and material specific finishing capabilities. A hybrid finishing process like rotational abrasive flow finishing (R-AFF) is one of modifications to raise the radial force and the polishing efficiency in AFM operation [1-3]. R-AFF had an approving performance in terms of better polishing and higher finishing rate, but this method may either rise machining cost or limit the machining shapes to process. Furthermore, Magneto-rheological abrasive flow finishing (MR-AFF) is also one of the improving actions as 
compared to the common AFM process [4,5]. In MR-AFF, the magnetic field applied to increase the abrasive forces, but this method was limited to the simple geometries of machining workpiece. Recently, a novel conception with inserting a mold core into a machining passageway to enhance the polishing rates was been developed. The results indicated that surface roughness of the cylindrical passageway notably decreased to $0.28 \mu \mathrm{m}$ Ra from an initial value of $1.8 \mu \mathrm{m}$ Ra after 5 working cycles [6]. Based on experimental study, it was observed that abrasive mesh size and percentage of abrasive concentration in media are the most significant parameters for material removal and improvement in surface roughness. Also viscosity of media was found as significant parameter for material removal for the considered size and shape of the workpiece. Moreover, a unique mechanism with a helical mold was created to build helical flowing paths. This design is an easier modification of mechanism with powerful rotation to raise the radial force in AFM process [7-9]. The experimental results indicated that the helical passageways performed better than the original passageways during AFM in terms of efficiency and creating polishing uniformity of the polygonal holes.

Abrasive media plays a major role in AFM because of its ability to precisely polish the selected surfaces along the media flow passageway. Thus, the investigation of different gels is an extremely importance in AFM field. Researchers have attempted to enhance the performance capabilities of AFM and also in development of superior and alternative to commercially available media. Davis et al. [10] investigated rheological study on different medium by homogeneously mixing polyborosiloxane (PBS) with silicon carbide (SiC) grits in varying proportion. Jain et al. [11] demonstrated the same by conducting AFF experiments using medium of different viscosities. Media usually contains two major roles as the carrier and the solid phase. The physical, chemical, material, and rheological characteristics significantly influence the overall performance of the AFM. Sinhg et al. [12] reported the work to model forces responsible for shearing of the roughness peaks during AFF process, and their correlation with rheological properties of the medium. It is the complex shear modulus of the medium which governs the magnitude of forces generated in AFF process by applying a MATLAB program. Wan et al. [13] performed the CFD simulation of the two-way AFF process during finishing of straight tubes with ellipsoidal cross sections. Final surface roughness of the workpiece is simulated and validated with the experimental result.

In this research, lower-cost and polymer based environmental friendly AFM media synthesis are developed to form polymer abrasive gels (PAGs) of various grades and upon trial on AFM setup. And an analytical model has been developed in order to understand the behaviour of motion of the different abrasive media by utilizing CFD-ACE ${ }^{+}$software; then, a series of AFM experiments were utilized to verify the simulated results.

\section{Materials and Methods}

\subsection{Gel Materials}

The most important factor which can affect the finishing result in AFM is the abrasive medium. In this study, a pure silicone rubber (P-Silicone) and silicone rubber with additives (A-Silicone) are chosen as the media. In general, P-Silicone is used to make the plastic mold in metal casting with low melting temperature. This kind of macromolecule gel belongs to high density visco-elastic rubber compound, mainly plays the role of mixed adhesive abrasive in mixed polymer gel and provides flow property, so that it can be deformed by different processing shapes. The composition of the polymer formula is as shown in Figure 1. Moreover, the actually manufacturing procedure of polymer silicon rubber is the first through the acidification process to destroy the molecular chain. Then, the gel medium is made through the high temperature synthesis of polymer compounds after 
several times repeatable processes. This material is a vinyl-based silicone rubber compounds with several hundred thousand molecules. Figure 2 shows that diagrams regarding (a) the transparent gel of P-Silicone and (b) the gel mixed with abrasive grains.<smiles>C[Si](C)(I)O[Al][Si](C)(C)[IH]O[Si](C)(C)[Si](C)(C)C</smiles>

Figure 1. Composition of the silicone polymer formula.

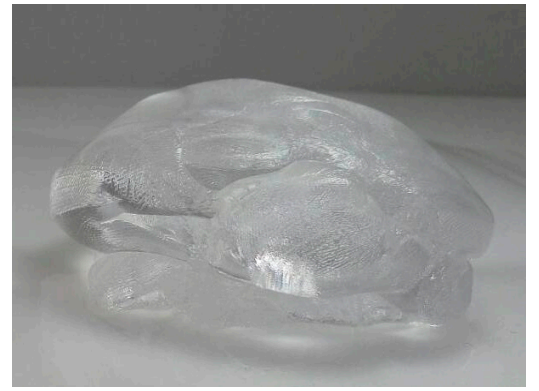

(a)

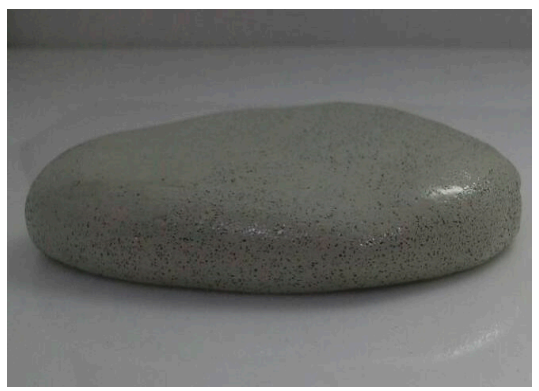

(b)

Figure 2. Diagrams regarding the transparent gel of P-Silicone and the gel mixed with abrasive grains. (a) P-silicone gel without abrasive; (b) P-silicone gel mixed with abrasive grains.

And A-Silicone is much like the material of the silly putty which is used for the toy clay. This material is also a vinyl-based silicone rubber compounds but being higher density with several million molecules. These silicone gels have lower flow property and do not stick on the workpiece surface after contact, so that they are the good abrasive media in AFM. And the complicated shape can be polished uniformly using this kind of silicone gels. Because the gels have good deformability, the abrasive particles must have high hardness to execute the polishing process. Figure 3 displays that diagrams regarding (a) the orange color gel of A-Silicone and (b) the polymer gel mixed with abrasive particles. Consequently, the silicon carbon $(\mathrm{SiC})$ is chosen as the main abrasive material and uniformly mixed with the silicone gel. This section may be divided by subheadings. It should provide a concise and precise description of the experimental results, their interpretation as well as the experimental conclusions that can be drawn.

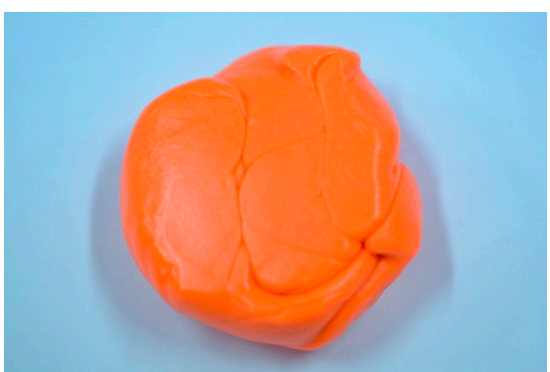

(a)

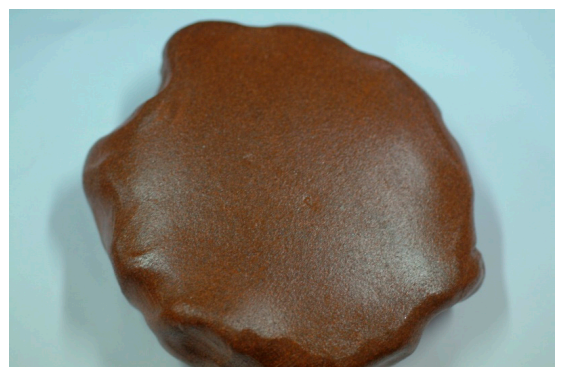

(b)

Figure 3. Diagrams regarding the orange color gel of A-Silicone and the gel mixed with abrasive grains. (a) A-silicone gel without abrasives; (b) A-silicone gel mixed with abrasive particles. 


\subsection{Materials Properties}

In this research, an analytical model has been developed in order to study the motion behaviour of the different abrasive media. However, the material properties of viscosity should be investigated before simulation to improve the accuracy of analysis. Therefore, a rheological device is used to determine the curve of shear rates relative to viscosities. Figure 4 shows the experimental data between the viscosities and the shear rates of the A-silicon medium with abrasive grains at a constant temperature of $27^{\circ} \mathrm{C}$. This flowing behavior of abrasive medium can be considered as a non-Newtonian fluid and shear thinning fluid from a macro perspective. Therefore, the power law is used to determine the relationships between viscosity and the shear rates. Gabriella et al., [14] discovered the mathematical expression of power law which is shown as Equation (1) according to the Ostwald-de Waele formulation, where $n$ represents the power law index and $n<1$ for the material of shear thinning fluid. In some materials, viscosity also decreases with increasing temperature. In ideal viscous materials, this phenomenon can be described by the Arrhenius Equation (2). Moreover, the function considers the relationships among viscosity, shear rates and temperature. The following mathematical Equation (3) describes a general model of the non-Newtonian power law to calculate the fluid viscosity of the abrasive medium:

$$
\begin{gathered}
\mu=K \dot{\gamma}^{(n-1)} \\
\mu=\mu_{0} e^{(B / T)} \\
\mu=K \mu_{0} e^{(B / T)} \dot{\gamma}^{(n-1)}
\end{gathered}
$$

The MATLAB software is applied to calculate and fit the power law curve. The material properties of viscosity coefficients can be calculated by the numerical method and are listed in Table 1. Furthermore, the experimental data takes the log of both $x$ and $y$; it describes a family of straight lines. Then, the MATLAB function of " polyfit " is used to find the slope and intercept. Finally, Figure 4 shows the curve fitting of the power law; the results can verify the consistency of both the experiment data and the MATLAB analytical curve.

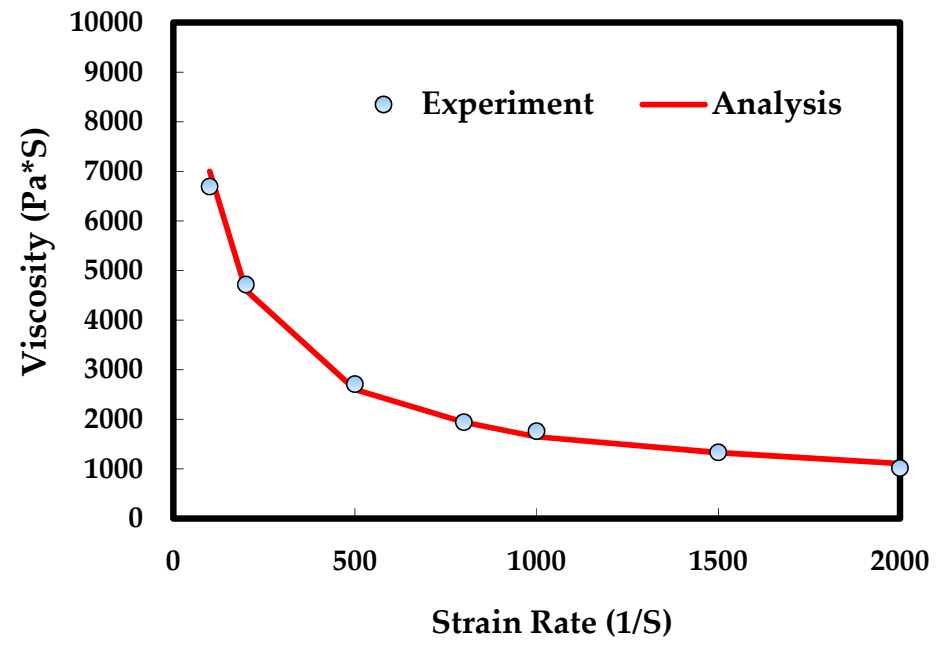

Figure 4. Diagram of viscosity v.s. shear rate. 
Table 1. Coefficient list for a power law

\begin{tabular}{lcccc}
\hline Matrix & $\mathrm{K}$ & $\mu_{0}$ & $\mathrm{~B}$ & $\mathrm{n}$ \\
\hline A-Silicone & 1 & 51000 & -0.002 & 0.379 \\
\hline
\end{tabular}

\subsection{AFM Set Up and Workpiece Materials}

In the traditional AFM process, deformable cutting tool known as AFM media is extruded over the surfaces to be finished because of imparting external pressure $P$. The medium motion reciprocates at velocity $V_{a}$ and exerts axial force $F_{a}$ on an abrasive particle along the axial direction of the workpiece. This demonstrates that the abrasive medium retains a unitary axial motion. It also exerts radial force $F_{r}$ on an abrasive particle interacting with the workpiece due to the visco-elastic character of the abrasive medium. The penetration depth of the abrasive grains in the workpiece surface depends on $F_{r}$ and the corresponding compressive strength of the abrasive medium, extrusion pressure and grain size. Then, axial force $F_{a}$ removes the material in the form of micro bits during the material removal process. A diagram of the velocity and force components during the AFM process is presented in Figure 5. In this present work, a novel mechanism with a helical passageway is developed to perform multiple flowing paths of abrasive medium by modifying AFM set-up. The diagram of the velocity and force components in AFM with a helical passageway is shown in Figure 6. In such a case, the helical motion in the polishing area exerts an additional tangential force $F_{t}$ along the tangential direction of workpiece; the following mathematical formula describing the total force $F_{c}$ of an abrasive particle as Equation (4) has been calculated. Resistance offered by the workpiece for removal of material in the form of microchips is expressed by $F_{S}$ as Equation (5). From a physical point of view, the material is removed in the form of microchips if $F_{c}>F_{S}$, and in some cases it may take place if $F_{c}=F_{S}$; then, the material removal just starts:

$$
\begin{aligned}
& F_{c}=\sqrt{F_{r}^{2}+F_{a}^{2}+F_{t}^{2}} \\
& F_{s}=\tau_{s} * A
\end{aligned}
$$

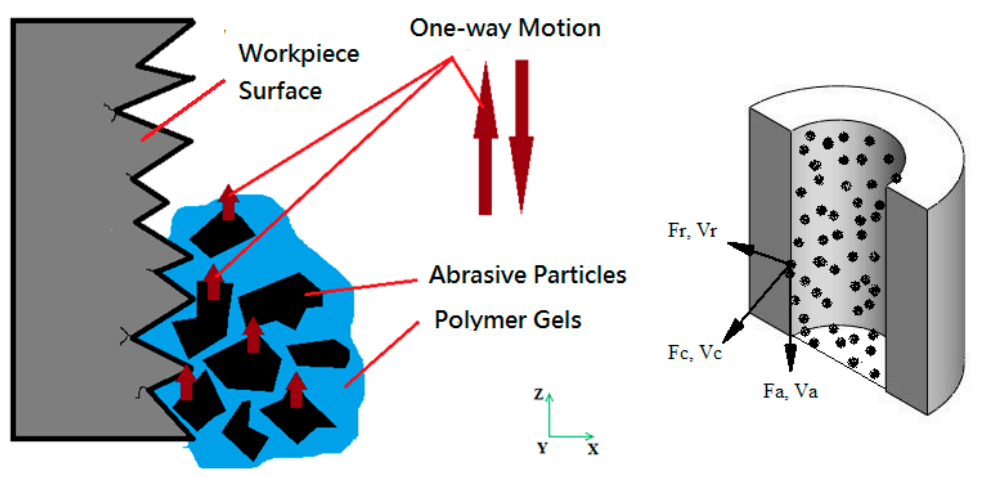

Figure 5. Diagram of the velocity and force components in traditional AFM. 
6 of 13

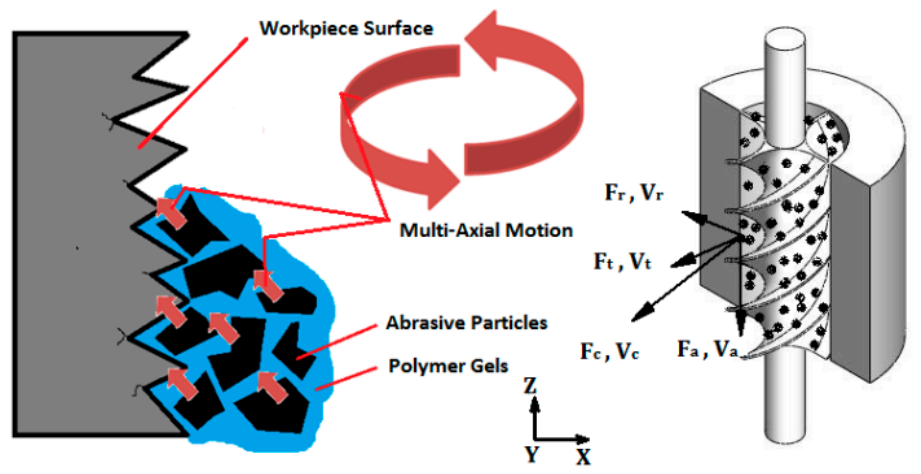

Figure 6. Diagram of the velocity and force components in AFM with a helical passageway.

This investigation focuses on increasing roughness improvement rate (RIR) of a radial distribution when polishing workpieces with A-silicon material. The mold steel SKD-11 is selected as workpiece material. It is widely used in the punching or injection molds. Batch complex-hole samples of SKD-11 steel are cut out using the WEDM process, in which the initially average SR of all these workpieces is approximately $0.65 \mu \mathrm{m}$ Ra after machining. The outer diameter of each complex-hole workpiece is $18.0 \mathrm{~mm}$, the inner diameter is $17.0 \mathrm{~mm}$, and the length is approximately $30.0 \mathrm{~mm}$. Figure 7 shows the diagrams regarding (a) the cutting part of a complex-hole specimen and (b) design parameters of a helical core.

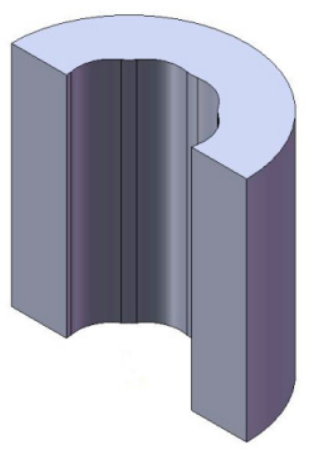

(a)

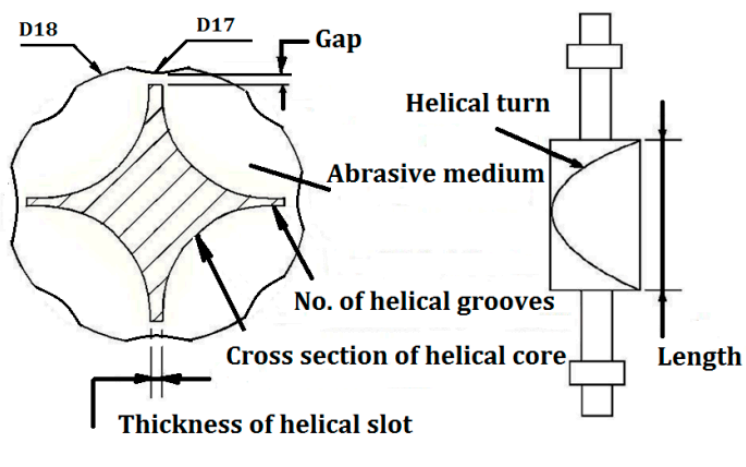

(b)

Figure 7. Diagrams of a complex-hole specimen, and cross-section of a helical core. (a) Cutting part of a complex-hole; (b) cross-section diagram of a helical core.

\section{Results and Discussion}

\subsection{Material Viscosities}

The rheological properties of the medium play an important role to find the good surface quality in AFM. In this experiment, a rheological apparatus is utilized to measure the medium viscosities when the abrasive concentration of the medium is $50 \%$. Figure 8 shows the effects of shear rates on medium viscosities. The result shows that the A-Silicone has higher viscosity than the P-Silicone in shear rate test. And the viscosities are decreased with increasing the shear rates both two silicon materials. It also shows that the high viscosity medium can make good elongation in the deformation test. 


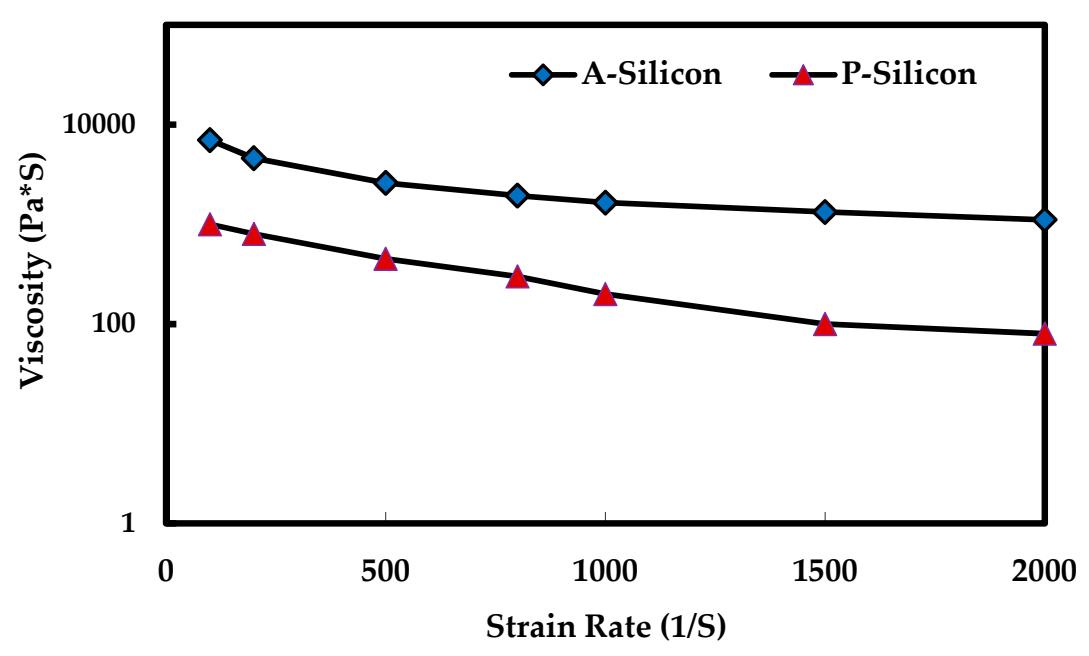

Figure 8. Diagram of the effect of strain rates on the viscosities for the different gels.

\subsection{Simulation Results of different Medium Properties}

This study has developed a numerical method to predict the flowing behaviours of the abrasive medium in complex holes before a series of AFM-related experiments. The material properties of viscosity coefficients can be calculated by the above-mentioned numerical method. Figure 9 shows the full modeling of a mesh diagram of the abrasive medium in a complex-hole passageway. Simulation results for velocities and strain rates of the abrasive medium are used to determine how these different medium properties affect the polishing precision during AFM.

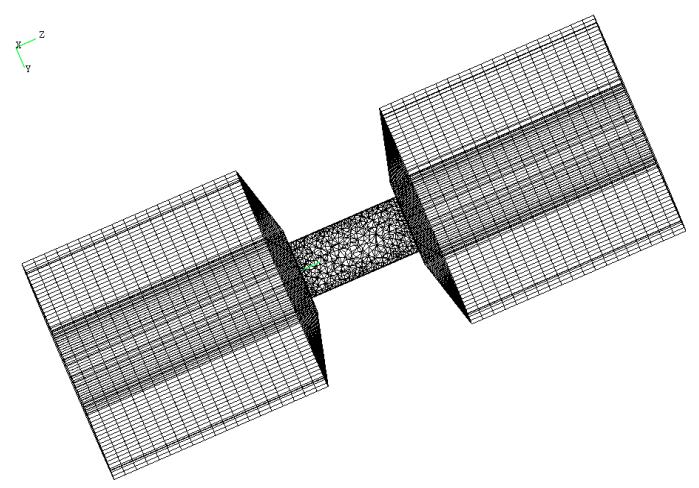

Figure 9. CFD modeling diagrams of the abrasive medium.

\subsubsection{Effects on velocity}

This subsection illustrates the axial motion of the abrasive media in a complex-hole polishing. The axial curves of three velocities with the different gels are given in Figure 10(a) and 10(b). For objective comparison, the measurement position is chosen to be $1 \mathrm{~mm}$ far away from the surface of the workpiece. The results indicate the velocity curves are near zero value on $\mathrm{x}$ and $\mathrm{y}$ directions, but $\mathrm{z}$ direction has a regular curve of velocity. The velocity distribution in figure 10 demonstrated the A-silicon and P-silicon with abrasive media retained a unitary axial motion. The result shows that the A-Silicone has lower flowing velocity than the P-Silicone in axial motion, when the viscosity of A-silicon is larger than that of P-silicon. 
Relatively, the contour distributions of radial velocity with the different gels are given in Figure 11(a) and 11(b). The result in radial motion also shows that the A-Silicone has lower flowing velocity than the P-Silicone in whole area of contour section.

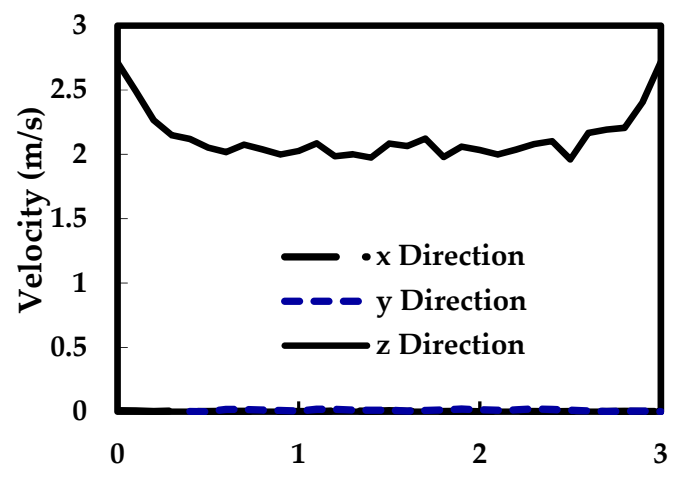

Axis Length of Complex-Hole (cm)

(a)

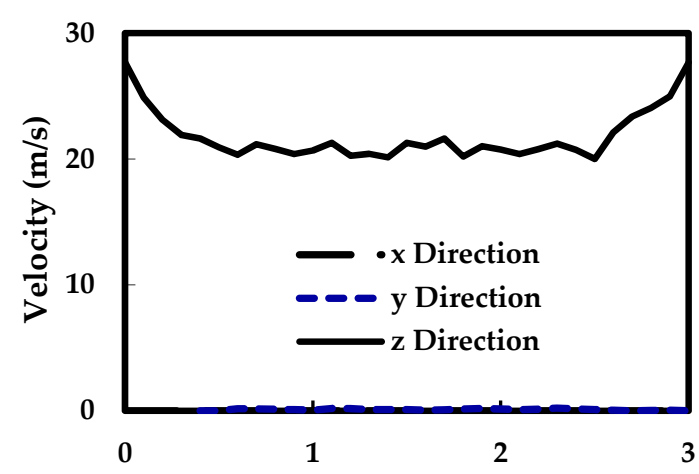

Axis Length of Complex-Hole (cm)

(b)

Figure 10. The simulation results of axial curve diagram for different silicon materials. (a) Velocity in axial direction (A-silicon); (b) Velocity in axial direction (P-silicon).

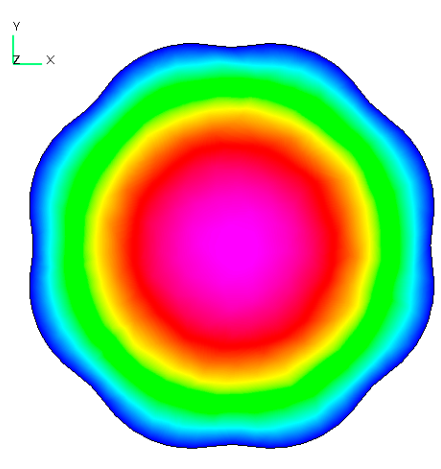

(a)
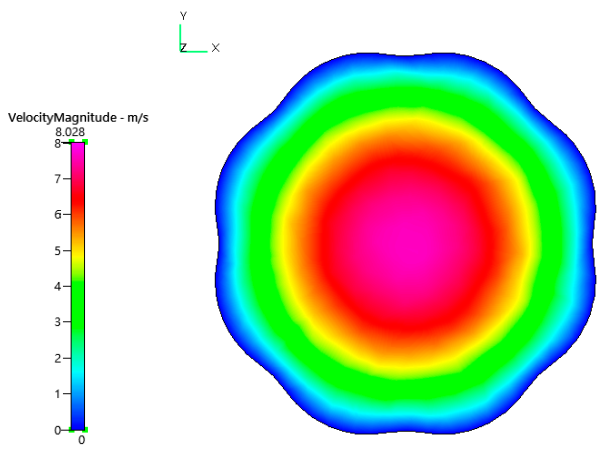

(b)

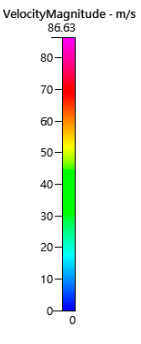

Figure 11. The simulation results of radial curve diagram for different silicon materials. (a) Velocity in radial direction (A-silicon); (b) Velocity in radial direction (P-silicon).

\subsubsection{Effects on strain rate}

Figure 12 shows the axial curve diagram of strain rates distribution. This simulation result indicates that the different deviation of strain rate distributes in entire complex-hole surface during axial motion, and predicts to reduce the uniformity precision of polishing complex holes. According to the figure, the strain rate of A-silicon gel is much smaller than that of P-silicon gel. Therefore, A-silicon gel has a large shear capacity and better material removal rate than P-silicon. Furthermore, A-silicon performed a low deviation of strain rate from peak to peak. In contrast, it shows the axial curve diagram of strain rates in the P-silicon, and the upper and lower limits of strain rates are widely distributed. Therefore, the simulated results indeed indicate that A-silicon predicts the enhancement of uniformity precision in AFM process. 


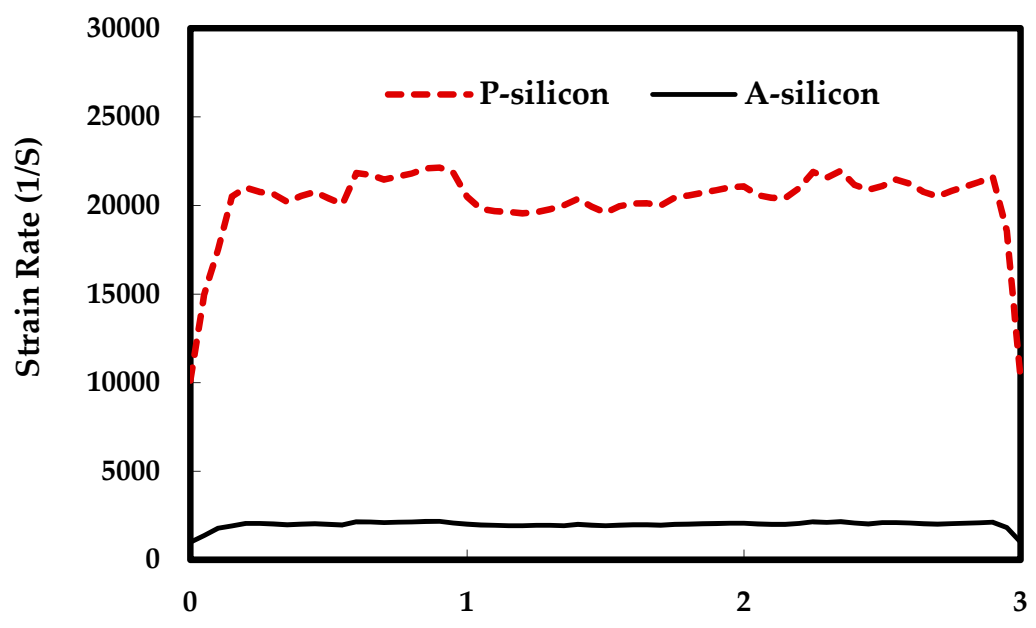

Axis Length of Complex-Hole (cm)

Figure 12. The simulation results of axial curve diagram for strain rate in different silicon materials.

\subsection{Durable Property of Abrasive Media}

The abrasive media must have stable chemical and physical properties, so that they are suitable for long duration of used in AFM. The goal is to verify the polishing effects for different passageways regarding material removal (MR) and RIR. To achieve the stated objective, material weight measurements and surface roughness measurements are taken to demonstrate the deviation for the items of MR and RIR in the radial direction after the AFM process. A series of experiments picked up eight positions in a radial surface to test the surface roughness during AFM. The RIR is defined as the following equation (6):

$$
\mathrm{RIR}=\frac{\mathrm{SR}_{\text {origin }}-\mathrm{SR}_{\text {polishing }}}{\mathrm{SR}_{\text {origin }}}
$$

Where SRorigin represents the original surface roughness before AFM, and SRpolishing describes the surface roughness after AFM polishing. Figure 13 represents the durable property of the same abrasive grain and concentration with the different gels. The effects of the number of up and down cycles on the surface roughness are been observed. It is illustrated that an increase in the cycles results in an associated decrease in the overall surface roughness. In general, the roughness is better with increasing number of up and down cycles. It is also shown that A-Silicone can polish a smoother surface than P-Silicone in AFM due to A-silicone has the higher viscosity and more materials to be removed. The medium temperature of both A-silicon and P-silicon is only risen 2 to 4 degrees Centigrade after 20 cycles in AFM. So the polishing effect is stable and a more precise surface can be obtained when A-Silicone is used as the medium. Figure 13 also displays the effects of the number of up and down cycles on RIR. It shows that the RIR is always maintained at $58 \%$ when the same A-Silicone is used to polish the workpieces after 20 cycling times. The RIR can also be maintained at a stable value of $35 \%$ if the same work is done by the P-Silicone. The reason is that A-Silicone and P-Silicone are flexible media; they can change their shape easily during the machining process. So the abrasive force is small and it reduces the particle abrasion in AFM. Furthermore, the new abrasives will always appear on the work surface when the abrasive medium changes its shape. Hence, no matter the A-Silicone or the P-Silicone is used, the durable property of these media is good to depend on. 
10 of 13

Figure 13. The durable property of the same abrasive media in different silicon materials.

\subsection{Polishing Effects of Different Passageways}

In this study, the task is to find the optimum conditions for different design parameters of a helical passageway, and machining parameters using the method of Taguchi reaction characteristics. Then, the effects of complex-hole with or without a helical core are studied. Based on our experimental result of passed database [9], it was observed that the optimal parameters of a helical passageway included a four-helix groove, a $0.5 \mathrm{~mm}$ gap, a $0.5 \mathrm{~mm}$ thickness of the helical slot, and a one helical spin to conduct the following experiments.

\subsubsection{Effects on Surface Roughness}

The subsection states the polishing effectiveness of abrasive medium with/without a helical core in polishing the complex-hole. Ten positions are picked during a series of experiments in a section to evaluate the surface roughness after AFM. Figure 14 presents the polishing effects on SR and MR after 20 AFM cycles in different passageways. This figure shows that a four-helix passageway performed better than a complex-hole passageway after AFM polishing in terms of efficiency; the RIR is only $58 \%$ in a complex-hole passageway, but the RIR can reach $84 \%$ in a four-helix passageway. This is largely owing to the abrasive medium in a complex-hole passageway only created unitary axial motion; that is why SR is slowly reduced in AFM. Relatively, the flowing paths in a four-helix passageway created dramatic variety when an abrasive medium is pushed through the passageway. Additionally, the MR of a four-helix passageway is also significantly better than a complex-hole passageway during AFM. Therefore, this study demonstrates that the effectiveness of a helical passageway obviously affected the average RIR and MR. 
11 of 13

Figure 14. Effects of surface roughness and material removal.

\subsubsection{Effects on Uniformity of Surface Roughness}

In this present study, surface roughness measurements of workpieces along the radial direction are led to verify the efficiency of the helical passageway during AFM polishing. Further, roughness uniformity (RU) is evaluated by measuring the changed deviation of the whole curve of surface roughness. The RU is defined by the deviation of surface roughness along the axial direction as shown in equation (7):

$$
\mathrm{RU}=S R_{\max }-S R_{\min }
$$

Where SRmax. represents the maximum surface roughness, and SRmin. describes the minimum surface roughness. Figure 15 shows the effect of different passageways on RU. According to the test results, the distribution of RU is $0.144 \mu \mathrm{m}$ Ra with a complex-hole passageway in AFM; but RU can reduce to $0.032 \mu \mathrm{m}$ Ra when a four-helix passageway is utilized. This study demonstrates the efficiency of a four-helix passageway in surface roughness uniformity based on calculating the percentage of improvement ratio around $78 \%$. Thus, these results indicate that surface of the complex holes are not uncommonly even in the radial direction. On the other hand, surface roughness is more smoothly when a four-helix core utilized as finishing passageway.

Figure15. Effects of different passageways on roughness uniformity. 


\subsubsection{Comparisons of SEM Pphotography on SR}

The AFM's ability to reduce surface roughness of workpiece is clearly demonstrated by above experimental results. Further, the surface characteristics of workpieces are illustrated herein to compare the variance at the same place before and after finishing. These figures are taken by the device of scanning electron microscope (SEM). Due to the discharge erosion effect, the WEDM surface is full of the craters. The surface is not smooth at all. Nevertheless, it is clearly visible that the surface characteristics of SEM micrography at 500X before and after finishing can be compared. Figure 16(a) presents the SEM micrography of an initial workpiece surface after WEDM machining, and Figure 16(b) displays the SEM micrography of a workpiece surface after a four-helix passageway in AFM polishing. The comparison result of SEM micrography shows that the even surface polished by AFM is great significance than the workpiece's surface by WEDM.

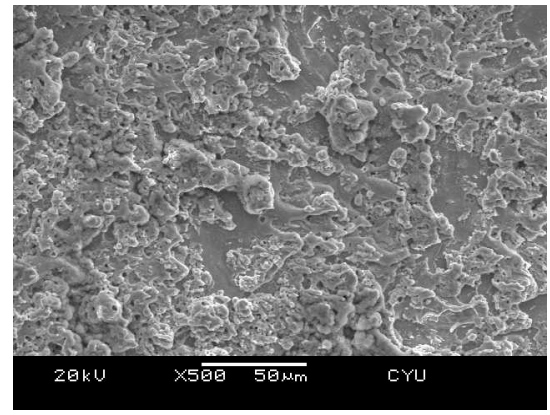

(a)

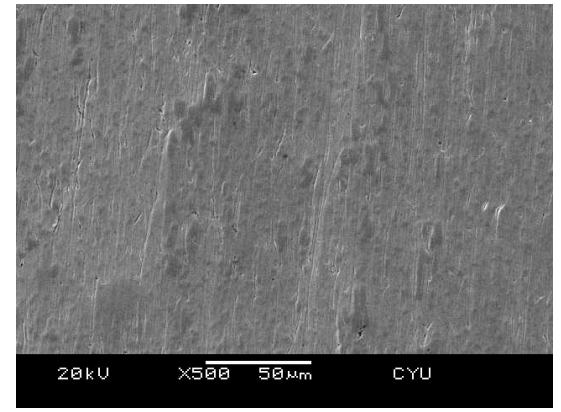

(b)

Figure 16. Surface characteristics of SEM micrography at 500X before and after AFM polishing. (a) Initial SEM after WEDM; (b) SEM after a helix core polishing.

\section{Conclusions}

Based on the foregoing results in investigating the flowing behaviours of the abrasive media in complex holes AFM polishing, the main conclusions are summarized as follows.

1. First, the result shows that the A-Silicone has higher viscosity and lower flowing velocity than the P-Silicone to result in a lower deviation of strain rate distribution. Therefore, the simulated results indeed indicate that A-silicon predicts the enhancement of uniformity precision in AFM process.

2. Next, the durable property of the same abrasive grain and concentration with the different gels is been studied, This results show that A-silicon performed better than P-silicon after AFM polishing in terms of efficiency; the RIR was only 35\% in P-silicon gels, but the RIR could reach $58 \%$ in A-silicon gels.

3. Moreover, experimental results of adopted a helical passageway show that SR significantly decreased from $0.65 \mu \mathrm{m} \mathrm{Ra}$ down to $0.104 \mu \mathrm{m} \mathrm{Ra}$, and calculating the percentage of improvement rate approximately $84 \%$ presented an excellent solution to improve surface precision in AFM processes.

Acknowledgments: The authors would like to thank the National Science Council of the Republic of China for financially supporting this research under Contract No. NSC 98-2221-E-231-007.

Author Contributions: A-Cheng Wang and Kuan-Yu Chen conceived of the idea for this research, designed the experiments, and reviewed this manuscript; Ken-Chuan Cheng performed the simulation study, analyzed the data and wrote the paper; Cheng-Chin Chien performed the experiments. 
Conflicts of Interest: The authors declare no conflict of interest.

\section{References}

1. Sankar, M.R.; Jain, V.K.; Ramkumar, J. Rotational abrasive flow finishing (R-AFF) process and its effects on finished surface topography. Int. J. Mach. Tools Manuf. 2010. Volume 50, pp. 637-650.

2. Das, M.; Jain, V.K.; Ghoshdastidar, P.S. The Out-of-Roundness of the Internal Surfaces of Stainless Steel Tubes Finished by the Rotational-Magnetorheological Abrasive Flow Finishing Process. Mater. Manuf. Process. 2011, Volume 26(8), pp. 1073-1084.

3. Sankar, M.R.; Jain, V.K.; Ramkumar, J. Nano-finishing of cylindrical hard steel tubes using rotational abrasive flow finishing (R-AFF) process. Int. J. Adv. Manuf. Technol. 2016. Volume 85, pp. 2179-2187, DOI 10.1007/s00170-015-8189-5.

4. Hong, K.P.; Cho, Y.K.; Shin, B.C.; Cho, M.W.; Choi, S.B.; Cho,W.S.; Jae,J.J. Magnetorheological (MR) Polishing of Alumina-Reinforced Zirconia Ceramics Using Diamond Abrasives for Dental Application. Mater. Manuf. Process. 2012, Volume 27(10), pp. 1135-1138.

5. Wang, A.C.; Tsai, L.; Liu, C.H.; Liang, K.Z.; Lee, S.J. Elucidating the Optimal Parameters in Magnetic Finishing with Gel Abrasive. Mater. Manuf. Process. 2011, Volume 26(5), pp. 786-791.

6. Wang, A.C.; Tsai, L.; Liang, K.Z.; Liu, C.H.; Weng, S.H. Uniform surface polished method of complex holes in abrasive flow machining. T. Nonferr. Metal. Soc. 2009, Volume 19, pp.250 257.

7. Lin, C.T.; Yang, L.D.; Chow, H.M. Study of magnetic abrasive finishing in free-form surface operations using the Taguchi method. Int. J. Adv. Manuf. Technol. 2007, Volume 34, pp. 122-130.

8. Wang, A.C.; Cheng, K.C.; Chen, K.Y.; Chien, C.C. Elucidating the optimal parameters of a helical passageway in abrasive flow machining. Int. J. Surf. Sci. Eng. 2015, Volume 9, Nos. 2/3, pp. 145-158.

9. Chen, K.Y.; Cheng, K.C. A study of helical passageways applied to polygon holes in abrasive flow machining. Int. J. Adv. Manuf. Technol. 2014, Volume 74, pp. 781-790, DOI 10.1007/s00170-014-5940-2.

10. Davies, P.J.; Fletcher, A.J. The assessment of the rheological characteristics of various polyborosiloxane/grit mixtures as utilized in the abrasive flow machining process. Proceedings of the Institution of Mechanical Engineers, 1995, Volume 209, pp. 409-418.

11. Jain, V.K.; Ranganatha, C.; Muralidhar, K. Evaluation of rheological properties of medium for AFM process. Mach. Sci. Tech. 2001, Volume 5 (2), pp. 151-170.

12. Singh, S.; Raj, A.S.A.; Sankar, M.R.; Jain, V.K. Finishing force analysis and simulation of nanosurface roughness in abrasive flow finishing process using medium rheological properties. Int. J. Adv. Manuf. Technol. 2016, Volume 85, pp. 2163-2178, DOI 10.1007/s00170-015-8333-2.

13. Wan, S.; Ang, Y.J.; Sato, T.; Lim, G.C. Process modeling and CFD simulation of two-way abrasive flow machining. Int. J. Adv. Manuf. Technol. 2014, Volume 71, pp. 1077-1086.

14. Gabriella B.; Janos K. Non-isothermal steady flow of power-law fluids between parallel plates. Int. J. Math. Mod. Meth. Appl. Sci. 2012, Issue 1, Volume 6, pp. 122-129. 